\title{
COMPORTAMENTO DE VARIEDADES E HÍBRIDOS DE BANANEIRA (Musa spp.), EM DOIS CICLOS DE PRODUÇÃO NO SUDOESTE DA BAHIA ${ }^{1}$
}

\author{
SÉRGIO LUIZ RODRIGUES DONATO², SEBASTIÃO DE OLIVEIRA E SILVA³, ORLANDO ANTÔNIO LUCCA FILHO ${ }^{4}$, \\ MARCELO BEZERRA LIMA5, HERBAT DOMINGUES ${ }^{6}$, JULIANA DA SILVA ALVES ${ }^{7}$
}

\begin{abstract}
RESUMO - O objetivo deste trabalho foi caracterizar e avaliar o comportamento de 13 genótipos (variedades e híbridos) de bananeira dos grupos genômicos AAA, AAAA, AAB e AAAB, em dois ciclos de produção, no sudoeste da Bahia, região de Guanambi, mediante o uso de descritores fenotípicos relevantes para a identificação e seleção de indivíduos superiores. Foram avaliadas as variedades Prata-Anã e Pacovan (AAB), Grande Naine e Nanicão (AAA), e os híbridos PA42-44, Preciosa, Japira, Pacovan Ken e ST12-31 (AAAB) e Ambrosia, Calipso, Bucaneiro e FHIA-02 (AAAA), selecionadas na Embrapa Mandioca e Fruticultura Tropical. Utilizou-se o delineamento em blocos casualizados, com 13 tratamentos, cinco repetições, parcelas constituídas por 20 plantas, seis úteis, e espaçamento de 3,00 m x 2,00 m. Foram considerados os caracteres: altura da planta, perímetro do pseudocaule, número de folhas vivas no florescimento e na colheita, número de dias do plantio ao florescimento e à colheita, peso do cacho e de pencas, número de frutos, peso, comprimento e diâmetro do fruto. A 'Grande Naine' e a 'Nanicão' destacaram-se nos caracteres peso do cacho e das pencas, número de frutos, peso e comprimento do fruto; o híbrido FHIA-02 apresentou o maior diâmetro do fruto nos dois ciclos de produção e a maior precocidade para florescimento e colheita no segundo ciclo; a 'Calipso' foi o mais tardia para colheita; o ST12-31 apresentou a maior altura; a variedade Prata-Anã destacou-se no caráter número de folhas vivas no florescimento e na colheita; os híbridos tipo prata, PA42-44, Pacovan Ken, Preciosa e Japira destacaram-se nos parâmetros relacionados à qualidade dos frutos quando comparados às suas genitoras 'PrataAnã' e 'Pacovan', respectivamente; houve incremento nos parâmetros que refletem o desenvolvimento vegetativo e a produtividade do primeiro para o segundo ciclo de produção em todos os genótipos avaliados.
\end{abstract}

Termos para indexação: genótipos, triplóides, tetraplóides, caracteres agronômicos, avaliação.

\section{BEHAVIOR OF BANANA VARIETIES AND HYBRIDS (Musa spp.), IN TWO PRODUCTION CYCLE IN THE SOUTHWEST OF BAHIA STATE}

\begin{abstract}
The objective of this work was to characterize and evaluate the behavior of 13 banana genotypes (varieties and hybrids) AAA, AAAA, AAB and AAAB genomic groups, in two production cycles in southwest Bahia, using relevant phenotypic descriptors for the identification and selection of superior individuals. The Prata Anã and Pacovan (AAB), Grande Naine and Nanicão (AAA) varieties and the hybrids PA42-44, Pacovan Ken, Japira, Preciosa and ST12-31 (AAAB) and Ambrosia, Calipso, Bucaneiro and FHIA-02 (AAAA), selected at Embrapa Cassava and Fruit Crops, were evaluated. The experimental design was in random blocks, with 13 treatments, five repetitions, 20 plants per plot, six useful and 3.00 $\mathrm{m} \times 2.00 \mathrm{~m}$ spacing. The following characteristics were considered: Plant height, pseudostem perimeter, number of live leaves during flowering and harvest, number of days from planting to flowering and until harvest, bunch and hand weight, number of fruits, weight, length and diameter of fruit. Grande Naine, and Nanicão stood out for the characteristics of bunch and hand weight, number of fruits, fruit weight and length; the FHIA-02 hybrid presented greater fruit diameter in both production cycles and the earliest flowering and harvest in the second cycle; Calipso was the latest for harvest; ST12-31 was the tallest in height; the Prata Anã variety stood out for number of live leaves during flowering and harvest; the Prata type hybrids, PA42-44, Pacovan Ken, Preciosa and Japira, stood out for the parameters related to fruit quality when compared to its genitors Prata Anã and Pacovan, respectively; there was an increment of the parameters that reflected vegetative growth and yield from the first to the second production cycle for all genotypes evaluated.
\end{abstract}

Index term: genotypes, triplets, agricultural characters.

A banana é considerada mundialmente um importante alimento, em razão da sua composição química e conteúdo em vitaminas e minerais, principalmente potássio, destacando-se dentre as fruteiras tropicais como a fruta mais consumida, e isso se dá tanto pela sua versatilidade em termos de modalidades de uso (processamento, frita, cozida, consumo in natura) quanto pelos seus caracteres de sabor, aroma, higiene e facilidade de consumo in natura. O consumo per capita nacional é em torno de $25 \mathrm{~kg} / \mathrm{hab}$./ano (Anuário..., 2003). A banana foi a fruta mais produzida no mundo, em 2004, ocupando uma área total de 4,54 milhões de hectares, em 128 países, produzindo 70,59 milhões de toneladas. Os principais países produtores são: Índia, Brasil, China, Equador e Filipinas, representando $58 \%$ da produção mundial de banana (FAO, 2004). A área plantada com banana na Bahia é 53,04 mil hectares (IBGE, 2005), sendo que 6,2 mil ha se encontram no sudoeste da Bahia, distribuída no municípios de Bom Jesus da Lapa (3.500 ha), Urandi (2.000 ha), Malhada (400 ha) e Guanambi (300 ha) (Codevasf, 2001).
Embora exista um número expressivo de variedades de banana no Brasil, quando se considera preferência dos consumidores, produtividade, tolerância a pragas, porte adequado, resistência à seca e ao frio, restam poucas variedades com potencial agronômico para utilização comercial. As mais difundidas no País são as bananas tipo prata (Prata, Pacovan e Prata-Anã), responsáveis por 60\% da área cultivada; a Maçã, a Mysore, as bananas tipo Cavendish (Nanica, Nanicão e Grande Naine), preferidas pelo mercado internacional, e as bananas tipo Terra (Terra e D'Angola) (Silva et al., 2000; 2002a), existindo ainda, outras variedades em menor proporção, como as do tipo Figo ou Bluggoe, as do tipo Caru e do tipo Ouro (Moreira, 1999).

Uma das estratégias para a solução dos problemas mencionados é o desenvolvimento de variedades resistentes a pragas, que sejam precoces, produtivas e aceitas pelo mercado, por meio de programas de melhoramento genético, bem como sua avaliação e caracterização em áreas de produção quando são comparadas às

\footnotetext{
(Trabalho 189/2004). Recebido: 21/12/2004. Aceito para publicação: 23/02/2006. Parte da Dissertação do primeiro autor, apresentada à Universidade Federal de Pelotas, para obtenção do Título de Mestre.

${ }^{2}$ Engenheiro Agrônomo, Mestre, Professor - EAFAJT, C.P. 009, CEP: 46.430-000, Guanambi-BA. E-mail: sergiodonato@eafajt.gov.br.

${ }^{3}$ Engenheiro Agrônomo, Doutor, Pesquisador-Embrapa Mandioca e Fruticultura, C.P.7, CEP:44.380-000, Cruz das Almas-BA. E-mail: ssilva@cnpmf.embrapa.br 4 Engenheiro Agrônomo, Doutor, Professor - UFPel / FAEM, C.P.354, CEP:96.001-970, Pelotas-RS. E-mail: luccafilho@ufpel.tche.br.

${ }^{5}$ Engenheiro Agrônomo, Mestre, Pesquisador - Embrapa Mandioca e Fruticultura, C.P.7, CEP:44.380-000, Cruz das Almas-BA. E-mail: mlima@cnpmf.embrapa.br

${ }^{6}$ Técnico em Agropecuária - EAFAJT, C.P. 009, CEP: 46.430-000. Guanambi-BA.

Graduanda em Agronomia, Escola de Agronomia, Universidade Federal da Bahia, Cruz das Almas-BA - jualvesagr@yahoo.com.br.
} 
cultivares tradicionais (Silva et al., 2000; 2002a; 2002b).

Esforços vêm sendo desenvolvidos para o melhoramento da bananeira a partir de germoplasma natural selecionado pelo homem. No entanto, reprodução vegetativa e um limitado número de acessos selvagens disponíveis resultam numa base genética estreita, o que representa um risco iminente, semelhante ao que ocorreu com a bananicultura latino-americana de exportação baseada na cultivar Gros Michel, suscetível ao mal-do-Panamá. Atualmente, a mesma bananicultura, corre risco semelhante, por se basear num clone tipo Cavendish, a variedade Grande Naine (Ortiz et al., 1995; Silva et al., 2002a).

O programa de melhoramento genético da bananeira no Brasil teve início em novembro de 1982, em Cruz das Almas-BA, baseando-se no subgrupo Prata. Desde o início, os genótipos selecionados foram avaliados em diversas regiões do Brasil (Silva et al., 2002a). Como resultados práticos destas avaliações até o momento, tem-se a recomendação dos híbridos PA12-03 (Pioneira), FHIA-18, SH36-40 (Prata Graúda), PV42-68 (Pacovan Ken), PV42-85 (Preciosa), PV42-142 (Japira), YB42-21 (Tropical), FHIA-01 (Maravilha), ST42-08 (Garantida), PC42-01 (Caprichosa) e das variedades Caipira, Nam (Prata Baby) e Thap Maeo.

Os caracteres normalmente estudados nas avaliações de genótipos são: ciclo da cultura, altura da planta, perímetro do pseudocaule, peso do cacho, número de frutos por cacho, comprimento e diâmetro dos frutos, descritores considerados relevantes para a identificação e a seleção de indivíduos superiores, mediante o uso de caracteres morfológicos relevantes que possam vir a ser recomendados para incorporação aos sistemas de produção do agricultor da região (Flores, 2000; Silva et al., 2000).

Dessa forma, este trabalho teve por objetivo caraterizar e avaliar o comportamento de 13 genótipos (variedades e híbridos) de bananeira de diferentes grupos genômicos, em dois ciclos de produção, nas condições agroclimático-ecológicas do sudoeste da Bahia, região de Guanambi.

O trabalho foi implantado em 2002, em Latossolo VermelhoAmarelo, distrófico, típico A fraco, textura média fase caatinga hipoxerófila, relevo plano a suave ondulado, na área experimental da Escola Agrotécnica Federal Antônio José Teixeira, localizada no
Município de Guanambi, com latitude de $14^{\circ} 13^{\prime} 30^{\prime \prime}$ sul, longitude de 42 $46^{\prime} 53^{\prime}$ " oeste de Greenwich, altitude de $525 \mathrm{~m}$ e com as médias anuais: precipitação de $663,69 \mathrm{~mm}$, temperatura média de $26^{\circ} \mathrm{C}$ e umidade relativa do ar de 64\% (Levantamento, 1979; Embrapa, 1999).

Foram avaliadas em dois ciclos de produção (2003-2004) as variedades tipo prata, Prata-Anã, Pacovan (AAB); tipo Cavendish, Grande Naine e Nanicão (AAA); os híbridos tipo prata PA42-44, Preciosa (PV42-85), Japira (PV42-142), Pacovan Ken (PV42-68) e ST12-31 (AAAB), geradas e selecionadas pela Embrapa Mandioca e Fruticultura Tropical; os tipos Gros Michel Ambrosia, Calipso e Bucaneiro (AAAA), e o híbrido tipo Cavendish FHIA-02 (AAAA), introduzidos da Jamaíca e de Honduras, respectivamente, e selecionados pela Embrapa (Tabela 1).

$\mathrm{O}$ experimento foi instalado com mudas micropropagadas, cedidas pela Embrapa Mandioca e Fruticultura Tropical, multiplicadas na Campo Biotecnologia em Cruz das Almas-BA, e transportadas para o local do experimento em embalagem tipo rocambole, onde foram plantadas em sacos de polietileno de $15 \mathrm{~cm}$ x $25 \mathrm{~cm}$, contendo substrato apropriado e aclimatadas em telado (sombrite $50 \%$ ), por cerca de 30 dias.

O sistema de irrigação utilizado em campo foi aspersão convencional fixo com aspersores subcopa, e o manejo de irrigação calculado pelo método do turno de rega pre-fixado, baseado nos caracteres do solo, condições atmosféricas e nas necessidades da bananeira. Os tratos culturais utilizados foram baseados em recomendações técnicas (Alves, 1997; Moreira, 1999) e as adubações realizadas mediante análises de solo e de folha.

Foram avaliados caracteres observados nos estádios de florescimento e colheita do cacho de cada planta útil, descritos a seguir: altura da planta (APL), expressa em centímetros; perímetro do pseudocaule (PPS), em centímetros; número de dias do plantio ao florescimento (NDF); número de folhas vivas no florescimento (NFF); número de dias do plantio à colheita do cacho (NDC); número de folhas vivas na colheita (NFC); peso do cacho (PCA) e peso das pencas (PPE), expressos em quilogramas; número de frutos por cacho (NFR); peso médio do fruto (PMF), determinado no fruto central da fileira externa de frutos da segunda penca, em gramas; comprimento externo do fruto

TABELA 1 - Descrição dos genótipos de bananeira avaliados na Escola Agrotécnica Federal Antônio José Teixeira, Guanambi-BA, 2004.

\begin{tabular}{|c|c|c|}
\hline GENÓTIPO & $\begin{array}{c}\text { GRUPO } \\
\text { GENÔMICO }\end{array}$ & DESCRIÇÃO \\
\hline Prata-Anã & $\mathrm{AAB}$ & $\begin{array}{l}\text { Variedade tipo Prata, mutante de Branca, porte baixo a médio, de baixa produtividade, suscetível às } \\
\text { sigatokas amarela e negra e ao mal-do-Panamá. }\end{array}$ \\
\hline Pacovan & $\mathrm{AAB}$ & $\begin{array}{l}\text { Variedade tipo Prata, mutante da Prata Comum, porte alto, de baixa produtividade, suscetível às } \\
\text { sigatokas amarela e negra e ao mal-do-Panamá. }\end{array}$ \\
\hline Grande Naine & AAA & $\begin{array}{l}\text { Variedade tipo Cavendish, mutante de Nanica, porte médio a baixo, de alta produtividade, suscetível } \\
\text { às sigatokas amarela e negra e resistente ao mal -do-Panamá. }\end{array}$ \\
\hline Nanicão & AAA & $\begin{array}{l}\text { Variedade tipo Cavendish, mutante de Nanica, porte médio, de alta produtivi dade, suscetível às } \\
\text { sigatokas amarela e negra e resistente ao mal-do-Panamá. }\end{array}$ \\
\hline PA42-44 & AAAB & Híbrido tipo Prata (Prata-Anã x M53), porte baixo a médio, resistente às sigatokas amarela e negra. \\
\hline Preciosa & AAAB & $\begin{array}{l}\text { Híbrido tipo Prata (Pacovan x M53), porte alto, resistente às sigatokas amarela, negra e mal -do- } \\
\text { Panamá.. Foi recomendado com o nome de Preciosa }\end{array}$ \\
\hline Pacovan Ken & AAAB & $\begin{array}{l}\text { Híbrido tipo Prata (Pacovan x M53), porte alto, resistentes às sigatokas amarela, negra e mal -do- } \\
\text { Panamá. Recomendado com o nome de Pacovan Ken }\end{array}$ \\
\hline Japira & AAAB & $\begin{array}{l}\text { Híbrido tipo Prata (Pacovan x M53), porte alto, resistente às sigatokas amarela, negra e mal -do- } \\
\text { Panamá. }\end{array}$ \\
\hline ST12-31 & $\mathrm{AAAB}$ & $\begin{array}{l}\text { Híbrido tipo Prata (Prata São Tomé x Lidi), porte alto, resistente à Sigatoka amarela e matdo-Panamá } \\
\text { suscetível à Sigatoka -negra. }\end{array}$ \\
\hline Ambrosia & AAAA & $\begin{array}{l}\text { Híbrido tipo Gros Michel, derivado de Highgate, porte médio a alto, resistente ao mal-do-Panamá e a } \\
\text { Sigatoka-negra. }\end{array}$ \\
\hline Bucaneiro & AAAA & $\begin{array}{l}\text { Híbrido tipo Gros Michel, derivado de Highgate, porte médio a alto, resistente ao mal-do-Panamá e a } \\
\text { Sigatoka-negra. }\end{array}$ \\
\hline Calipso & AAAA & $\begin{array}{l}\text { Híbrido tipo Gros Michel, derivado de Highgate, porte médio a alto, resistente ao mal-do-Panamá e a } \\
\text { Sigatoka-negra. }\end{array}$ \\
\hline FHIA-02 & AAAA & $\begin{array}{l}\text { Híbrido tipo Cavendish (Williams x } \mathrm{SH} 33-93 \text { ), porte baixo, resistente às sigatokas negra e amarela, } \\
\text { suscetível ao mal-do-Panamá, introduzido da FHIA }{ }^{1} \text {. }\end{array}$ \\
\hline
\end{tabular}


TABELA 2 - Médias dos caracteres observados na época do florescimento de 13 genótipos de bananeira, em dois ciclos de produção. GuanambiBA, 2004 .

\begin{tabular}{|c|c|c|c|c|c|c|c|c|}
\hline \multirow[t]{3}{*}{ Genótipos } & \multicolumn{8}{|c|}{ Caracteres ${ }^{1}$} \\
\hline & \multicolumn{2}{|c|}{ APL $(\mathrm{cm})$} & \multicolumn{2}{|c|}{ PPS $(\mathrm{cm})$} & \multicolumn{2}{|c|}{ NFF (un) } & \multicolumn{2}{|c|}{ NDF (dias) } \\
\hline & $1^{\mathrm{o}}$ & $2^{\circ}$ & $1^{\circ}$ & $2^{\circ}$ & $1^{\mathrm{o}}$ & $2^{\mathrm{o}}$ & $1^{\mathrm{o}}$ & $2^{o}$ \\
\hline ST12-31 & $350,62 \mathrm{a}$ & $497,10 \mathrm{a}$ & $79,08 \mathrm{a}$ & $103,68 \mathrm{a}$ & $12,20 \mathrm{~d}$ & $15,07 \mathrm{c}$ & $247,90 \mathrm{c}$ & $466,39 \mathrm{~b}$ \\
\hline Pacovan Ken & $343,62 \mathrm{a}$ & $493,93 \mathrm{a}$ & $72,44 \mathrm{c}$ & $94,27 \mathrm{c}$ & $13,10 \mathrm{~d}$ & $15,93 \mathrm{~b}$ & $246,22 \mathrm{c}$ & $429,18 \mathrm{~d}$ \\
\hline Japira & $326,20 \mathrm{~b}$ & $472,08 \mathrm{~b}$ & $68,38 \mathrm{~d}$ & $91,94 \mathrm{c}$ & $13,00 \mathrm{~d}$ & $13,92 \mathrm{c}$ & $242,96 \mathrm{c}$ & $423,31 \mathrm{~d}$ \\
\hline Pacovan & $322,78 \mathrm{~b}$ & $444,26 \mathrm{c}$ & $69,56 \mathrm{~d}$ & $84,71 \mathrm{~d}$ & $16,46 \mathrm{~b}$ & $13,78 \mathrm{c}$ & $224,88 \mathrm{~d}$ & $390,98 \mathrm{e}$ \\
\hline Preciosa & $313,38 \mathrm{c}$ & $454,26 \mathrm{c}$ & $70,02 \mathrm{~d}$ & $92,40 \mathrm{c}$ & $12,72 \mathrm{~d}$ & $13,76 \mathrm{c}$ & $237,18 \mathrm{c}$ & $412,44 \mathrm{~d}$ \\
\hline Ambrosia & $285,72 \mathrm{~d}$ & $373,50 \mathrm{~d}$ & $74,66 \mathrm{~b}$ & $97,61 \mathrm{~b}$ & $10,86 \mathrm{f}$ & $13,82 \mathrm{c}$ & $277,50 \mathrm{a}$ & $445,70 \mathrm{c}$ \\
\hline Calipso & $284,94 \mathrm{~d}$ & $383,88 \mathrm{~d}$ & $76,50 \mathrm{~b}$ & $104,24 \mathrm{a}$ & $10,54 \mathrm{f}$ & $14,06 \mathrm{c}$ & $282,36 \mathrm{a}$ & $493,10 \mathrm{a}$ \\
\hline Bucaneiro & $278,44 \mathrm{~d}$ & $363,66 \mathrm{e}$ & $75,48 \mathrm{~b}$ & $98,01 \mathrm{~b}$ & $11,58 \mathrm{e}$ & $14,03 \mathrm{c}$ & $275,78 \mathrm{a}$ & $468,85 \mathrm{~b}$ \\
\hline Nanicão & $265,50 \mathrm{e}$ & $324,98 \mathrm{f}$ & $72,06 \mathrm{c}$ & $89,05 \mathrm{~d}$ & $12,92 \mathrm{~d}$ & $15,72 b$ & $276,70 a$ & $483,99 \mathrm{a}$ \\
\hline PA42-44 & $245,12 \mathrm{f}$ & $353,20 \mathrm{e}$ & $70,80 \mathrm{c}$ & $92,38 \mathrm{c}$ & $14,74 \mathrm{c}$ & $14,60 \mathrm{c}$ & $224,66 \mathrm{~d}$ & $390,57 \mathrm{e}$ \\
\hline Grande Naine & $244,54 \mathrm{f}$ & $300,46 \mathrm{~g}$ & $73,88 \mathrm{~b}$ & $87,99 \mathrm{~d}$ & $13,26 \mathrm{~d}$ & $16,00 \mathrm{~b}$ & $265,86 \mathrm{~b}$ & $470,97 \mathrm{~b}$ \\
\hline Prata-Anã & $243,36 \mathrm{f}$ & $359,03 \mathrm{e}$ & $75,48 \mathrm{~b}$ & $101,30 \mathrm{a}$ & $18,76 \mathrm{a}$ & $18,81 \mathrm{a}$ & $225,48 \mathrm{~d}$ & $419,91 \mathrm{~d}$ \\
\hline FHIA-02 & $229,02 \mathrm{~g}$ & $321,60 \mathrm{f}$ & $67,60 \mathrm{~d}$ & $85,49 \mathrm{~d}$ & $12,54 \mathrm{~d}$ & $13,34 \mathrm{c}$ & $242,78 \mathrm{c}$ & $373,98 \mathrm{e}$ \\
\hline CV (\%) & 2,65 & 3,78 & 2,42 & 3,55 & 4,22 & 6,28 & 3,26 & 3,44 \\
\hline
\end{tabular}

${ }^{1}$ APL: Altura da planta (cm); PPS: Perímetro do pseudocaule (cm); NFF: Número de folhas vivas no florescimento (unidade); NDF: Número de dias do plantio ao florescimento (dias).

(CEF), medido na curvatura externa do fruto central da segunda penca, em centímetros; diâmetro ou calibração lateral do fruto (DLF), em milímetros, medido na parte mediana, no sentido do comprimento do fruto central da segunda penca.

O delineamento experimental foi o de blocos casualizados, com 13 tratamentos e cinco repetições, constituindo assim 65 unidades experimentais. Cada parcela foi constituída por 20 plantas, sendo seis úteis, e no espaçamento de $3,0 \mathrm{~m}$ x 2,0 m. A variedade Prata-Anã, difundida na região, foi utilizada como bordadura para o experimento. Os dados foram submetidos à análise de variância, e as médias foram agrupadas pelo critério de Scott \& Knott, a 5\% de probabilidade, devido ao número elevado de tratamentos.

A altura da planta variou de 350,62 cm (ST12-31) a 229,02 cm (FHIA-02) no primeiro ciclo de produção e de 497,10 cm (ST12-31) a $300,46 \mathrm{~cm}$ (Grande Naine) no segundo ciclo. Houve a formação de sete agrupamentos, em função da alta variabilidade genética para este caráter existente entre os diversos genótipos (Tabela 2). A diferença percentual entre dois grupos subseqüentes variou de 7,97\% entre o grupo 5 e 6 a $3,42 \%$ entre o grupo 2 e 3 no primeiro ciclo e variou de $15,90 \%$ entre o grupo 3 e 4 a 4,62\% entre o grupo 2 e 3 no segundo ciclo.

A altura da planta é um descritor importante do ponto de vista fitotécnico e de melhoramento, pois influi nos aspectos de densidade de plantio e manejo da cultura, interferindo na produção (Belalcázar Carvajal, 1991).

O perímetro do pseudocaule apresentou variação de $79,08 \mathrm{~cm}$ (ST12-31) a 67,60 cm (FHIA-02). Os genótipos que apresentaram maior e menor altura, ST12-31 e FHIA-02, apresentaram, respectivamente, maior e menor perímetro do pseudocaule. No segundo ciclo de produção, o genótipo com maior perímetro do pseudocaule foi o híbrido Calipso, com 104,24 cm, que não diferiu de ST12-31 nem de Prata-Anã. Para o mesmo caráter, o menor valor correspondeu à variedade Pacovan, 84,71 cm, não diferindo de Nanicão, Grande Naine e FHIA 02. Foram identificados quatro grupos para este caráter nos dois ciclos de produção (Tabela 2).

O tombamento de plantas e/ou a quebra do pseudocaule pela ação dos ventos estão relacionados com elevado porte, reduzido perímetro do pseudocaule e produção de cachos grandes (Teixeira, 2001).

O número de folhas vivas no florescimento apresentou variação de 18,76 (Prata-Anã) a 10,54 (Calipso) com a formação de seis agrupamentos no primeiro ciclo (Tabela 2). No segundo ciclo de produção, a variedade Prata-Anã apresentou também o maior número de folhas vivas na época do florescimento $(18,81)$, sendo que o genótipo com menor valor para este caráter foi o híbrido FHIA-02, com 13 folhas.
Houve formação de três agrupamentos (Tabela 2). Este descritor é importante na avaliação de cultivares, pois poderá influenciar no desenvolvimento do cacho, o qual dependerá diretamente da taxa de fotossíntese da planta (Alves, 1997).

O genótipo mais precoce para florescimento foi o Híbrido PA4244, com 224,66 dias, e o mais tardio foi o híbrido Calipso, com 282,36 dias. Os genótipos foram divididos em quatro agrupamentos do mais precoce para o mais tardio (Tabela 2). O híbrido Calipso foi também, no segundo ciclo de produção, o genótipo mais tardio para florescimento, com 493 dias, e o FHIA-02 o mais precoce, com 374 dias. Os genótipos para este caráter foram divididos em cinco agrupamentos, no segundo ciclo de produção (Tabela 2).

Dentre os caracteres observados na época do florescimento, os que expressam o vigor, altura da planta e perímetro do pseudocaule, apresentaram incremento no segundo ciclo em relação ao primeiro para todos os genótipos avaliados, como era de se esperar, sendo o maior incremento para altura da planta verificado na variedade Prata-Anã (32\%) e o menor na Nanicão (11\%). Para o perímetro do pseudocaule, o maior incremento foi observado no híbrido Calipso (36\%) e o menor na variedade Grande Naine (16\%). Já para o número de folhas vivas avaliado na época do florescimento, houve decréscimo do primeiro para o segundo ciclo para os genótipos PA42-44 e Pacovan, e incremento para os demais.

Os descritores peso do cacho e das pencas, embora expressem diretamente a produtividade, não podem ser considerados isoladamente na escolha de uma cultivar, pois outros caracteres relacionados aos frutos, como peso, comprimento, diâmetro, sabor e resistência ao despencamento, devem ser considerados neste processo (Silva et al., 2002b).

Os descritores peso do cacho e peso das pencas apresentam estreita relação entre si, sendo que o peso do cacho variou de $32,42 \mathrm{~kg}$ para a variedade Grande Naine a 15,82 kg para o Híbrido ST12-31, e o peso das pencas teve variação de $29,28 \mathrm{~kg}$ para a variedade Grande Naine a $13,82 \mathrm{~kg}$ para o híbrido Japira, identificando-se assim três agrupamentos no primeiro ciclo (Tabela 3). Já, no segundo ciclo de produção, o peso do cacho variou de 44,17 kg para a variedade Nanicão a 20,16 kg para Pacovan, e o peso das pencas de 40,54 kg para a variedade Nanicão a 17,40 kg para a Pacovan, observando-se quatro agrupamentos (Tabela 3). Os genótipos do grupo 3 apresentaram produção de cacho $45,03 \%$ e $46,11 \%$ inferior aos do grupo 1 , no primeiro e segundo ciclos, respectivamente. Com relação ao peso das pencas, diferença percentual entre os grupos 1 e 3 foram, respectivamente, de $46,93 \%$ e $48,68 \%$ para o primeiro e segundo ciclos. 
TABELA 3 - Médias dos caracteres observados na época da colheita de 13 genótipos de bananeira, em dois ciclos de produção. Guanambi-BA, 2004.

\begin{tabular}{|c|c|c|c|c|c|c|c|c|c|c|}
\hline \multirow[t]{3}{*}{ Genótipos } & \multicolumn{10}{|c|}{ Caracteres ${ }^{1}$} \\
\hline & \multicolumn{2}{|c|}{ PCA (kg) } & \multicolumn{2}{|c|}{ PPE (kg) } & \multicolumn{2}{|c|}{ NFR (un) } & \multicolumn{2}{|c|}{ NFC (un) } & \multicolumn{2}{|c|}{ NDC (dias) } \\
\hline & $1^{\mathrm{o}}$ & $2^{o}$ & $1^{\mathrm{o}}$ & $2^{\circ}$ & $1^{\mathrm{o}}$ & $2^{\circ}$ & $1^{0}$ & $2^{o}$ & $1^{\circ}$ & $2^{o}$ \\
\hline Grande Naine & $32,42 \mathrm{a}$ & $43,24 \mathrm{a}$ & $29,28 \mathrm{a}$ & $39,78 \mathrm{a}$ & $165,84 \mathrm{a}$ & $199,20 \mathrm{a}$ & $10,10 \mathrm{~b}$ & $9,81 \mathrm{~b}$ & $359,90 \mathrm{~b}$ & $581,02 \mathrm{a}$ \\
\hline Nanicão & $30,12 \mathrm{a}$ & $44,17 \mathrm{a}$ & $27,02 \mathrm{a}$ & $40,54 \mathrm{a}$ & $158,02 \mathrm{~b}$ & $208,45 \mathrm{a}$ & $10,06 \mathrm{~b}$ & $9,83 \mathrm{~b}$ & $366,84 \mathrm{a}$ & $592,82 \mathrm{a}$ \\
\hline FHIA-02 & $24,00 \mathrm{~b}$ & $34,69 \mathrm{~b}$ & $21,28 \mathrm{~b}$ & $30,69 \mathrm{~b}$ & $132,20 \mathrm{c}$ & $174,88 \mathrm{~b}$ & $8,52 \mathrm{c}$ & $9,62 \mathrm{~b}$ & $358,56 \mathrm{~b}$ & $510,29 \mathrm{~d}$ \\
\hline Bucaneiro & $22,90 \mathrm{~b}$ & $38,46 \mathrm{~b}$ & $19,94 \mathrm{~b}$ & $34,93 \mathrm{~b}$ & $127,60 \mathrm{c}$ & $175,05 \mathrm{~b}$ & $9,00 \mathrm{c}$ & $8,46 \mathrm{c}$ & $370,90 \mathrm{a}$ & $580,53 \mathrm{a}$ \\
\hline Calipso & $21,28 \mathrm{~b}$ & $35,57 \mathrm{~b}$ & $19,06 \mathrm{~b}$ & $32,25 \mathrm{~b}$ & $130,54 \mathrm{c}$ & $177,37 \mathrm{~b}$ & $7,38 \mathrm{~d}$ & $8,41 \mathrm{c}$ & $374,54 \mathrm{a}$ & $596,56 \mathrm{a}$ \\
\hline Ambrosia & $20,22 \mathrm{~b}$ & $34,72 b$ & $17,96 \mathrm{~b}$ & $30,82 \mathrm{~b}$ & $124,82 \mathrm{c}$ & $171,75 \mathrm{~b}$ & $8,74 \mathrm{c}$ & $8,48 \mathrm{c}$ & $374,18 \mathrm{a}$ & $557,73 \mathrm{~b}$ \\
\hline Prata-Anã & $18,88 \mathrm{c}$ & $28,01 \mathrm{c}$ & $16,52 \mathrm{c}$ & $24,99 \mathrm{c}$ & $133,78 \mathrm{c}$ & $172,76 \mathrm{~b}$ & $11,88 \mathrm{a}$ & $12,01 \mathrm{a}$ & $362,46 \mathrm{~b}$ & $548,42 \mathrm{~b}$ \\
\hline PA42-44 & $18,14 \mathrm{c}$ & $26,14 \mathrm{c}$ & $15,96 \mathrm{c}$ & $22,82 \mathrm{c}$ & $102,26 \mathrm{~d}$ & $125,14 \mathrm{c}$ & $9,94 \mathrm{~b}$ & $10,22 \mathrm{~b}$ & $347,14 \mathrm{c}$ & $528,50 \mathrm{c}$ \\
\hline Pacovan & $17,80 \mathrm{c}$ & $20,16 \mathrm{~d}$ & $15,54 \mathrm{c}$ & $17,40 \mathrm{~d}$ & $102,42 \mathrm{~d}$ & $106,56 \mathrm{~d}$ & $10,70 \mathrm{~b}$ & $9,62 \mathrm{~b}$ & $360,04 \mathrm{~b}$ & $524,48 \mathrm{c}$ \\
\hline Pacovan Ken & $17,16 \mathrm{c}$ & $22,81 \mathrm{~d}$ & $14,84 \mathrm{c}$ & $19,85 \mathrm{~d}$ & $88,68 \mathrm{e}$ & $107,57 \mathrm{~d}$ & $9,48 \mathrm{~b}$ & $9,18 \mathrm{c}$ & $360,72 \mathrm{~b}$ & $549,18 \mathrm{~b}$ \\
\hline Preciosa & $16,34 \mathrm{c}$ & $22,32 \mathrm{~d}$ & $14,02 \mathrm{c}$ & $19,19 \mathrm{~d}$ & $86,50 \mathrm{e}$ & $110,00 \mathrm{~d}$ & $7,72 \mathrm{~d}$ & $8,63 \mathrm{c}$ & $360,36 \mathrm{~b}$ & $533,71 \mathrm{c}$ \\
\hline Japira & $16,18 \mathrm{c}$ & $23,20 \mathrm{~d}$ & $13,82 \mathrm{c}$ & $20,26 \mathrm{~d}$ & $87,20 \mathrm{e}$ & $113,24 \mathrm{~d}$ & $9,14 \mathrm{c}$ & $8,34 \mathrm{c}$ & $362,66 \mathrm{~b}$ & $555,26 \mathrm{~b}$ \\
\hline ST12-31 & $15,82 \mathrm{c}$ & $22,22 \mathrm{~d}$ & $13,92 \mathrm{c}$ & $19,75 \mathrm{~d}$ & $104,12 \mathrm{~d}$ & $122,76 \mathrm{c}$ & $9,54 \mathrm{~b}$ & $7,86 \mathrm{c}$ & $365,44 \mathrm{a}$ & $593,80 \mathrm{a}$ \\
\hline CV (\%) & 11,75 & 10,39 & 11,48 & 10,68 & 5,07 & 6,02 & 8,03 & 9,34 & 1,70 & 2,45 \\
\hline
\end{tabular}

'PCA: Peso do cacho (kg); PPE: Peso das pencas (kg); NFR: Número de frutos (unidade); NFC: Número de folhas vivas na colheita (unidade); NDC: Número de dias do plantio à colheita (dias).

A produtividade, de modo geral, variou com o grupo genômico (potencial genético) e o porte das plantas. Os genótipos AAA e AAAA foram mais produtivos que $\mathrm{AAB}$ e $\mathrm{AAAB}$, o que era esperado, uma vez que os tipo Cavendish e Gros Michel são potencialmente mais produtivos que os tipo Prata; entretanto, independentemente do grupo, os genótipos de porte mais baixo foram mais produtivos que os de porte mais alto.

O primeiro ciclo não é o momento oportuno para analisar o peso do cacho na maioria dos genótipos, uma vez que o caráter pode aumentar do primeiro para o segundo ciclo da cultura (Silva et al., 2002b), o que é corroborado por este trabalho. Embora algumas cultivares, como a Grande Naine, possam produzir mesmo no primeiro ciclo cachos grandes, com 32,42 kg, observados neste trabalho, e 30,30 kg obtidos por Donato et al. (2003).

O número de frutos por cacho variou de 165,84 na variedade Grande Naine a 86,50 frutos no híbrido Preciosa, o que permitiu a formação de cinco agrupamentos (Tabela 3). Os genótipos com maior e menor número de frutos por cacho no segundo ciclo de produção foram, respectivamente, as variedades Nanicão, com 208,45 e Pacovan, com 106,56 frutos, o que levou à formação de quatro agrupamentos (Tabela $3)$.

O primeiro ciclo também não deve ser considerado conclusivo para analisar o desempenho de genótipos quanto ao número de frutos, pois há uma tendência de elevação nos ciclos posteriores no valor desse caráter (Flores, 2000; Silva et al., 2002b), como também foi obtido neste trabalho. Um incremento no número de frutos entre os ciclos foi também observado por Donato et al. (2003), para a variedade Grande Naine, em experimento realizado no município de Guanambi-BA.

O número de folhas vivas na colheita, variou de 11,88 na variedade Prata-Anã, a 7,38 folhas no híbrido Calipso, o que permitiu a identificação de quatro agrupamentos (Tabela 3 ). No segundo ciclo de produção, o caráter em questão variou de 12,0 para a variedade PrataAnã a 7,9 no híbrido ST12-31, dividindo os genótipos em três grupos (Tabela 3).

Os valores do número de folhas vivas na colheita, obtidos neste trabalho para a maioria dos genótipos avaliados, foram elevados quando comparados a outros estudos (Ledo et al., 1997; Flores, 2000; Silva et al., 2000), provavelmente devido à não-ocorrência de Sigatoka de forma epidêmica na região de Guanambi (Donato, 2003), doença que causa grande destruição foliar e, conseqüentemente, redução de produção.

No primeiro ciclo de produção, identificaram-se três agrupamentos distintos com relação ao número de dias para a colheita. O caráter variou de 374,54 dias para o híbrido Calipso a 347,14 dias para o híbrido PA42-44. Já no segundo ciclo de produção, o número de dias permitiu a formação de quatro agrupamentos e variou de 596,56 dias para o híbrido Calipso a 510,29 dias para o FHIA-02 (Tabela 3).

Para o peso do fruto, os genótipos foram distribuídos em dois agrupamentos. Este caráter variou de 200,44 g para a variedade Grande Naine a 140,76 g para o híbrido ST12-31. O peso do fruto, no segundo ciclo de produção, variou de 218,38 g no híbrido Bucaneiro a 157,09 g na variedade Pacovan, permitindo a formação de três agrupamentos distintos (Tabela 4). O peso do fruto é um caráter importante para os trabalhos de melhoramento, sendo que não pode ser considerado isoladamente, mas sim associado a outros componentes que refletem a qualidade dos frutos, como o comprimento e o diâmetro do fruto. $\mathrm{O}$ peso de frutos em híbridos é sempre superior ao das cultivares genitoras femininas (Silva et al., 2002a).

O comprimento do fruto variou de $23,14 \mathrm{~cm}$ para a variedade Grande Naine a $16,58 \mathrm{~cm}$ para a Prata-Anã, e de $24,13 \mathrm{~cm}$ para a variedade Nanicão a 17,40 cm para o híbrido ST12-31, no primeiro e segundo ciclos, respectivamente, formando, em ambos os ciclos, cinco agrupamentos (Tabela 4).

O caráter diâmetro lateral do fruto variou de $35,98 \mathrm{~mm}$ no híbrido FHIA-02 a 31,94 mm para o híbrido Japira. Assim como no primeiro ciclo, o híbrido FHIA-02 apresentou maior diâmetro do fruto no segundo ciclo de produção, 37,29 mm. Já, o genótipo que apresentou menor diâmetro do fruto nesta fase foi o híbrido Pacovan Ken (33,40 mm). Identificaram-se dois agrupamentos distintos nos dois ciclos (Tabela 4).

Analisando o comprimento e o diâmetro lateral dos frutos para as bananas tipo Prata e tomando como base sua classificação, apresentada em manual ilustrado (Ministério da Integração Nacional, 2000), pode-se afirmar, com relação a estes caracteres, que todos os genótipos tipo Prata avaliados enquadram-se no tipo exportação, com exceção do Japira no primeiro ciclo, que apresentou o diâmetro lateral abaixo do exigido. O diâmetro do fruto é normalmente usado para indicar o ponto de colheita. Frutos que não atingem o comprimento e o diâmetro adequados são descartados na comercialização (Jaramillo, 1982; Soto Ballestero, 1992; Moreira, 1999).

Dentre os caracteres observados na época da colheita, houve incremento do primeiro para o segundo ciclo para todos os genótipos avaliados, no peso do cacho, peso das pencas, número de frutos e comprimento do fruto. Entretanto, para o número de folhas na época da colheita, peso médio e diâmetro lateral do fruto, houve decréscimo para alguns genótipos. Para o peso do cacho, o genótipo que apresentou maior incremento, foi o híbrido Ambrosia ( $72 \%$ ), e o menor foi a variedade Pacovan (13\%). O peso das pencas mostrou maior incremento do 
TABELA 4 - Médias dos caracteres observados na época da colheita (caracteres relacionados à qualidade dos frutos) de 13 genótipos de bananeira, em dois ciclos de produção. Guanambi-BA, 2004.

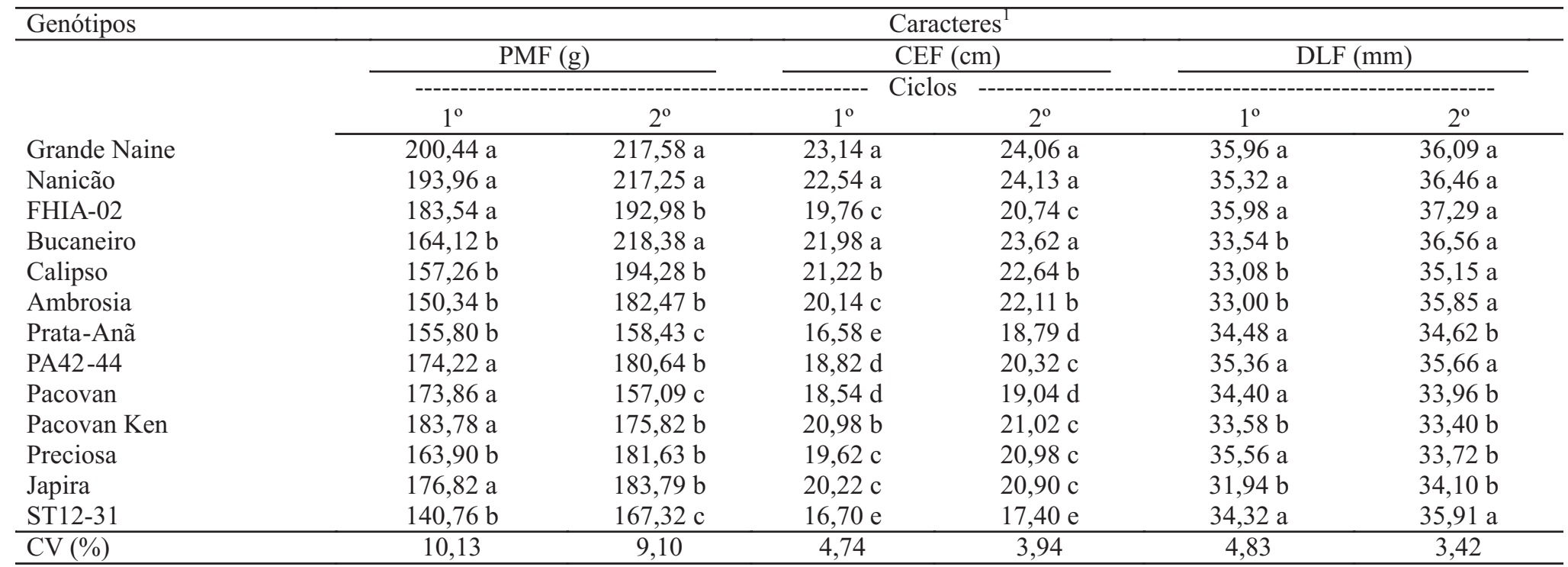

'PMF: Peso médio do fruto (g); CEF: Comprimento externo do fruto (cm); DLF: Diâmetro lateral do fruto (mm).

primeiro para o segundo ciclo no híbrido Bucaneiro (75\%) e menor na variedade Pacovan (12\%). Com relação ao número de frutos por cacho, o maior incremento do primeiro para o segundo ciclo ocorreu no genótipo Ambrosia (37\%), e o menor na variedade Pacovan (4\%).

Quando se avalia apenas um ciclo de produção, a produtividade é estimada com base no peso do cacho e na população de plantas. No caso de mais de um ciclo, a produtividade é calculada considerando três componentes, quais sejam, peso do cacho, população de plantas por hectare e duração do ciclo (Robinson \& Nel, 1988). Assim, após dois ciclos de produção para peso do cacho, expressa em t/ha/ ano, tem-se que o genótipo menos produtivo foi o híbrido tipo Prata de porte alto ST12-31, com 38,4 t/ha/ano, e os mais produtivos foram as variedades do subgrupo Cavendish, Grande Naine com 78,1 t/ha/ano e Nanicão com 75,2 t/ha/ano em razão do maior potencial genético dessas variedades.

Houve incremento em produtividade baseada no caráter peso do cacho do primeiro para o segundo ciclo, para todos os genótipos estudados, como era de se esperar, sendo o maior incremento verificado no híbrido Ambrosia (41\%), e o menor na variedade Pacovan (11,60\%).

O híbrido PA42-44 destacou-se na qualidade dos frutos, embora apresente maior despencamento em relação à genitora. O híbrido ST12-31 apresentou caracteres indesejáveis, como porte alto, produtividade baixa e frutos curtos. Os híbridos Pacovan Ken, Preciosa e Japira superaram a sua genitora (Pacovan) na qualidade dos frutos. Embora o FHIA-02 tenha apresentado precocidade, alta produtividade, frutos grandes, planta com porte médio e resistente a ventos fortes, o fruto tem a polpa muito mole, despenca com relativa facilidade e a planta é suscetível ao mal-do-Panamá, o que foi observado neste ensaio. Os genótipos tipo Gros Michel foram bastante produtivos nos dois ciclos, mas possuem pencas imbricadas, frutos com pedicelos curtos, o que dificulta as operações de retirada de pencas e confecção de buquês, além de elevado despencamento.

Os resultados obtidos neste trabalho qualificam os genótipos avaliados como promissores, sendo necessárias, entretanto, avaliações em um número maior de ciclos de produção e melhor aferição com vistas à aceitação dos mercados consumidores.

Os híbridos avaliados apresentam desempenho equivalente ou superior às variedades correspondentes.

Permite-se qualificar como promissores para serem incorporados aos sistemas de produção do agricultor da região, os híbridos tipo Prata PA42-44, Pacovan Ken, Preciosa e Japira e tipo Gros Michel Bucaneiro, Ambrosia e Calipso.

As condições do sudoeste da Bahia, são favoráveis para a expressão da maioria dos caracteres avaliados, evidenciando adequação ambiental e/ou de manejo.

\section{REFERÊNCIAS}

ALVES, E. J. (Org.). A cultura da banana: aspectos técnicos socioeconômicos e agroindustriais. Brasília: SPI / Cruz das Almas: Embrapa-CNPMF, 1997. 585 p.

BELALCÁZAR CARVAJAL, S. L. El cultivo del plátano em el trópico. Cali: Impresora Feriva, 1991.376 p.

BELING, R.R. et al. Anuário brasileiro da fruticultura. Santa Cruz do Sul: Editora Gazeta Santa Cruz, 2003.136 p.

BRASIL. Ministério da Integração Nacional. Banana: Minas Gerais. Brasília, DF: Secretaria de Infraestrutura Hídrica. Departamento de projetos Especiais - DPE. 8p. (FrutiSéries, 6).

CODEVASF. CENSO FRUTÍCOLA 2001 Disponível em: <http:// www.codevasf.gov.br/ censofrutícola >. Acesso em: 10 mar. 2003.

DONATO, S. L. R.; SILVA, S. de O. e; PASSOS, A. R.; LIMANETO, F. P.; LIMA, M. B. Avaliação de variedades e híbridos de bananeira sob irrigação. Revista Brasileira de Fruticultura, Jaboticabal, v. 25, n. 2, p. 348-351, 2003.

DONATO, S. L. R. Comportamento de variedades e híbridos de bananeira (Musa spp.), em primeiro ciclo de produção no Sudoeste da Bahia, Região de Guanambi. 2003. 115f. Dissertação (Mestrado em Ciência e Tecnologia de Sementes) - Faculdade de Agronomia "Eliseu Maciel", Universidade Federal de Pelotas, Pelotas, RS, 2003.

EMBRAPA. Centro Nacional de Pesquisa de Solos. Sistema brasileiro de classificação de solos. Rio de Janeiro: Embrapa Solos, 1999. 412p.

FAO. Food and Agricultural Organization. Disponível em: $<$ http:// apps.fao.org/page/ collections>. 2004. Acesso em: 29 maio. 2004.

FLORES, J. C. de O. Avaliação de cultivares e híbridos de bananeira (Musa spp.) em quatro ciclos de produção em Cruz das Almas-BA. 2000. 109 f. Dissertação (Mestrado em Fruticultura Tropical) - Escola de Agronomia, Universidade Federal da Bahia, Cruz das AlmasBA, 2000.

IBGE. Produção agrícola municipal. Disponível em: $<\mathrm{http}: / /$ www.sidra.ibge.gov.br>. Acesso em: 21 jun. 2005.

JARAMILLO, R. C. Las principales características morfológicas del fruto de banano, variedade Cavendish Gigante (Musa AAA) em Costa Rica. Upeb-Impretex, 1982. 42 p.

LEDO, A. da S.; LEDO, F. J. da SILVA; SILVA, S. de O. e. Avaliação de cultivares de banana em Rio Branco-Acre. Rio Branco: Embrapa - CPAF/AC, 1997. 16 p. (Boletim de Pesquisa, 15).

LEVANTAMENTO exploratório: reconhecimento dos solos da margem 
direita do Rio São Francisco. Estado da Bahia. Recife: Embrapa: SNLCS: SUDENE, 1979. v.2.1.296 p. (Boletim Técnico, 52).

MOREIRA, R. S. Banana, teoria e prática de cultivo. 2.ed. São Paulo: Fundação Cargill, 1999. CD-ROM.

ORTIZ, R.; FERRIS, R. S. B.; VUYLSTEKE, D. R. Banana and plantain breeding. In: GOWEN, S. (Ed.). Bananas and plantains. London: Chapman \& Hall, 1995. cap.5, p. 110-146.

ROBINSON, J. C.; NEL, D.J. Plant density studies with banana (cv. Williams) in a subtropical climate. I. Vegetative morphology, phenology and plantation microclimate. Journal of Horticultural Science, Ashford, v.63, n.2, p.303-313, 1988.

SILVA, S. de O. e; ALVES, E. J.; LIMA, M. B.; SILVEIRA J. R. da S. Bananeira. In: BRUCKNER C. H. (Ed.). Melhoramento de fruteiras tropicais. Viçosa: UFV, 2002a. p. 101-157.

SILVA, S.O.; FLORES, J. C.; LIMANETO, F. P. Avaliação de cultivares e híbridos de bananeira em quatro ciclos de produção. Pesquisa Agropecuária Brasileira, Brasília, v. 37, n. 11, p. 1.567-1.574, 2002 b. SILVA, S. O.; ROCHA, S. A.; ALVES, E. J.; CREDICO, M. DI.; PASSOS, A. R. Caracterização morfológica e avaliação de cultivares e híbridos de bananeira. Revista Brasileira de Fruticultura, Jaboticabal - SP, v. 22, n. 2, p. 161-169, agosto, 2000.

SOTO BALLESTERO, M. Bananos: cultivo e comercializacion. 2. ed. San José, Costa Rica: Litografia e Imprensa LIL, 1992. 674 p.

TEIXEIRA, L. A. J. Cultivares de bananeira. In: RUGGIERO, C. (Coor.). Bananicultura. Jaboticabal: FUNEP, 2001.p. 150-170. 